\title{
A New Monotonicity Index for Fuzzy Rule-based Systems
}

\author{
${ }^{1}$ Lie Meng Pang, ${ }^{* 1}$ Kai Meng Tay, ${ }^{2}$ Chee Peng Lim \\ ${ }^{1}$ Faculty of Engineering, Universiti Malaysia Sarawak, Sarawak, Malaysia \\ ${ }^{2}$ Centre for Intelligent Systems Research, Deakin University, Australia. \\ Corresponding author:*kmtay@feng.unimas.my
}

\begin{abstract}
A search in the literature reveals that mathematical conditions (usually sufficient conditions) for the Fuzzy Inference System (FIS) models to satisfy the monotonicity property have been developed. A monotonicallyordered fuzzy rule base is important to maintain the monotonicity property of an FIS. However, it may difficult to obtain a monotonically-ordered fuzzy rule base in practice. We have previously introduced the idea of fuzzy rule relabeling to tackle this problem. In this paper, we further propose a monotonicity index for the FIS system, which serves as a metric to indicate the degree of a fuzzy rule base fulfilling the monotonicity property. The index is useful to provide an indication whether a fuzzy rule base should (or should not) be used in practice, even with fuzzy rule relabeling. To illustrate the idea, the zero-order Sugeno FIS model is exemplified. We add noise as errors into the fuzzy rule base to formulate a set of non-monotone fuzzy rules. As such, the metric also acts as a measure of noise in the fuzzy rule base. The results show that the proposed metric is useful to indicate the degree of a fuzzy rule base fulfilling the monotonicity property.
\end{abstract}

Keywords- Fuzzy inference system; monotonicity index ; fuzzy rule base.

\section{INTRODUCTION}

In general, a monotonically-ordered fuzzy rule base is important to maintain the monotonicity property of a Fuzzy Inference System (FIS) model [1-4]. However, it is difficult to ensure that users are able to obtain a monotonicallyordered fuzzy rule in practice [5-6]. In our previous investigations [5-6], the idea of fuzzy rule relabeling was introduced. Fuzzy rule relabeling attempts to relabel the non-monotone fuzzy rules, and produces a set of monotonically-ordered fuzzy rule base. In this paper, noise (hereafter refer to as monotone noise) is defined as errors in the fuzzy rule base that leads to the formation of a set of nonmonotone fuzzy rules [5-6]. Fuzzy rule relabeling can then be used to pre-process (or filter) monotone noise in the fuzzy rule base; therefore serving as a monotone noise removal method.

One key question is how monotone noise in a fuzzy rule base can be measured and analyzed. The idea of signal-tonoise ratio, which can be used to compare the level of a desired signal to the level of background noise, is not new, especially in signal processing [7] and image processing [8]. In this paper, we introduce a new monotonicity index for FISs. This monotonicity index provides a metric to measure the extent a fuzzy rule base satisfies the monotonicity property, or free from monotone noise. Such metric is important owing to a number of reasons: (1) it allows expert knowledge (in a form of a fuzzy rule base) for a monotone FIS model to be analyzed; (2) it gives an indication on the level of monotone noise in a fuzzy rule base that interferes with information pertaining to the subject of interest; (3) it provides an indication whether a fuzzy rule base should (or should not) be used in practice, even with fuzzy rule relabeling; and (4) it can potentially be used to analyze monotone noise of hierarchical FIS models.

The idea of a monotonicity test for precise data [9] or a function [10] is not new. In [9], a measure of the monotonicity property for a set of precise data was proposed. It attempts to give an indication to what extent independent variables follow a monotone relationship with response variables in a set of data [9]. In our previous work [10], a monotonicity test for a function was proposed. The monotonicity test [10] gives an approximate indication whether a function (e.g. an FIS model) satisfies the monotonicity property. Besides that, it is also worth mentioning that Spearman's rank correlation coefficient [12] was proposed to assess how well the relationship of two precise variables can be described using a monotone function. Differing from the above approaches, the failure to obtain a set of monotonically-ordered fuzzy rule base is viewed as a source of monotone noise in this paper, which needs to be measured and analyzed. Such assumption was adopted in [11] for the case of precise data points.

In this paper, a procedure to compute the monotonicity index of two fuzzy rules and a fuzzy rule base is firstly explained. We focus on the zero-order Sugeno FIS model. In addition, the use of the monotonicity index for hierarchical FIS models is further discussed. This paper contributes towards formulating a monotonicity index for the FIS models. The proposed procedure is evaluated using simulated examples.

The organization of this paper is as follows. The background of the Sugeno FIS model and its monotonicity property are presented in Section II. In Section III, the proposed monotonicity test for two fuzzy rules is described. A monotonicity test for measuring the monotone degree of a fuzzy rule base is presented in Section IV. In Section V, the potential of applying the proposed 